\author{
反应型比例苂光探针检测多巴胺

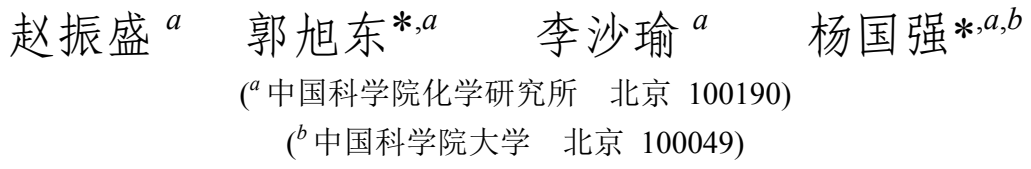

\begin{abstract}
摘要 多巴胺是一种重要的神经递质, 可调控脑功能和人体的行为反应, 因此对多巴胺浓度的检测是非常重要的. 合 成了基于萠的苂光探针羟基(米基)(萠-1-基)硼烷(HMPB)，能够在缓冲溶液中对多巴胺快速响应，并且在较低浓度下都 有响应，同时具有很好的选择性. 为生物体内检测多巴胺提供了一种潜在的高灵敏性、高选择性的检测方法.

关键词＼cjkstart多巴胺；荧光探针；嗍化物；荧光光谱法；高选择性
\end{abstract}

\title{
Reaction-Based Ratiometric Fluorescence Probes For Dopamine Detection
}

\author{
Zhao, Zhensheng $^{a} \quad$ Guo, Xudong*,a ${ }^{*}$ Li, Shayu ${ }^{a} \quad$ Yang, Guoqiang*,a,b \\ ( ${ }^{a}$ Institute of Chemistry Chinese Academy of Sciences, Beijing 100190) \\ ( ${ }^{b}$ University of Chinese Academy of Sciences, Beijing 100049)
}

\begin{abstract}
Dopamine (DA) is one of neurotransmitters in the human central nervous system and plays a very important role on human behavior and brain function. It is necessary to detect dopamine and determine its concentration in organism. Of all the dopamine detection methods reported, fluorescence probes exhibit high efficiency, considerate specifity and potential for real-time detection. Considering the high quantum field and strong trend to form an excimer of pyrene fluorophore, a fluorescent probe hydroxy(mesityl)(pyren-1-yl)borane (HMPB) based on pyrenyl boron compound was designed, synthesized and utilized in dopamine detection. In the phosphate buffer solution (PBS) with the aid of surfactant decyltrimethylammonium bromide, the water-insoluble HMPB could show two fluorescent bands: a monomer band of peaked at $388 \mathrm{~nm}$ and an excimer band peaked at $484 \mathrm{~nm}$. The solubility of HMPB improved after addition of DA because it could react with HMPB to form a new compound HMPB-DA and it is better to dissolve in water. The excimer pyrenyl, therefore, gradually dissociated to generate monomer pyrenyl. Accordingly, as the addition of DA, fluorescent intensity at $484 \mathrm{~nm}\left(I_{484 \mathrm{~nm}}\right)$ decreased, while intensity at $388 \mathrm{~nm}\left(I_{388 \mathrm{~nm}}\right)$ increased. Within $10 \mathrm{~min}$, the fluorescence intensity reached saturation and the ratio of $I_{388 \mathrm{~nm}}$ to $I_{484 \mathrm{~nm}}$ have great changed after adding dopamine. HMPB exhibited significant fluorescent response when the concentration range of DA is $10 \mathrm{nmol} / \mathrm{L}$ to $600 \mathrm{nmol} / \mathrm{L}$ in $10 \mathrm{~min}$, which matched the physiologic concentration of DA. The ratio of $I_{388 \mathrm{~nm}}$ to $I_{484 \mathrm{~nm}}$ could be utilized to determine the concentration of dopamine. The detection limit of HMPB was as low as 14.6 $\mathrm{nmol} / \mathrm{L}$. HMPB showed no response to common amino acids, saccharides, proteins, ions, catecholamin and other bioactive molecules, even if the interference molecules were at high concentrations. Meanwhile, HMPB can be used to detect dopamine in urine of organisms. With fast response, high sensitivity and accuracy, HMPB represented considerable potential to act as a DA detector in physiological environment, which could become a promising tool in biochemistry, molecular biology and diagnostics investigation.
\end{abstract}

Keywords dopamine; fluorescent probe; boron compound; spectrofluorimetry; high selectivity

\section{1 引言}

多巴胺(DA)是一种儿茶酚胺类神经递质, 对脑功 能和行为反应起到重要作用, 诸如感觉、信息传导和成 㒣等神经过程都受其影响 ${ }^{[1]}$. 体液(例如, 尿液、血浆和 中枢神经系统的细胞外液)中的多巴胺浓度是许多疾病 的诊断指标 ${ }^{[2]}$. 例如, 亨廷顿病可增加多巴胺的分泌, 造成体内多巴胺浓度过高, 进而引发代谢能力衰竭, 甚 至致命; 此外, 某些疾病会使体内多巴胺浓度过低, 继 而引发肌肉失控、注意力下降等症状, 甚至导致帕金森
病. 因此对人体中多巴胺浓度的检测具有重要的科学意 义和诊断学意义.

已有多种多巴胺检测方法见诸报道, 如电致化学发 光法 ${ }^{[3]}$ 、电化学法 ${ }^{[4]}$ 、毛细管电泳 ${ }^{[5]}$ 、比色法 ${ }^{[6]}$ 、紫外可 见光谱法 ${ }^{[7]}$ 、高效液相色谱法 ${ }^{[8]}$ 和流动注射分析法 ${ }^{[9]}$ 等. 而这些方法都有一定的局限性，例如，人体中的尿酸和 抗坏血酸在很大程度上限制了电化学方法的选择性. 与 之相比，苂光光谱法具有成本低、操作简单、高重复性 和灵敏度高等一系列优点，因此已被越来越多地用于多 巴胺检测 ${ }^{[10]}$. 如, 吕式课题组利用(3-氨基丙基)三甲氧 基硅(APTMS)包覆的氧化锌量子点在水中和血清中检

*E-mail: gqyang@iccas.ac.cn

Received April 6, 2016; published May 12, 2016.

Supporting information for this article is available free of charge via the Internet at http:/sioc-journal.cn.

Project supported by the National Natural Science Foundation of China (Nos. 21206122, 21233011).

项目受国家自然科学基金(Nos. 21206122, 21233011)资助. 
测多巴胺, 在 $0.05 \sim 10.0 \mu \mathrm{mol} / \mathrm{L}$ 范围内, 探针的荧光强 度与多巴胺浓度成线性关系, 检出限低达 $12 \mathrm{nmol} / \mathrm{L}^{[10 \mathrm{c}]}$; 钟新华课题组利用基于腺苷包覆 $\mathrm{Cd} / \mathrm{ZnS}$ 量子点的苂光 传感器尿样中检测多巴胺, 检出限为 $29.3 \mathrm{nmol} / \mathrm{L}^{[10 \mathrm{~b}]}$. 但是, 生命体系中多巴胺浓度一般为 $26 \sim 40 \mathrm{nmol} / \mathrm{L}$, 甚至更低 ${ }^{[11]}$. 因此, 发展灵敏性更好、选择性更好的多 巴胺荧光探针仍是一项巨大的挑战.

萠是一种量子产率较高的苂光团, 且易于形成激基 缔合物结构. 利用其单体与激基缔合物的发光光谱不 同, 可以构造比例荧光型探针. 比例荧光探针与传统的 荧光发光( “Turn-on” )型探针相比, 由于其具有自参比 特征, 检测的准确度更高, 且容易避免激发光源的影响. 因此, 本文主要介绍了一种用于检测多巴胺的反应型比 例苂光探针: 羟基(米基)(萠-1-基)嗍烷(HMPB). HMPB 是一种油溶性萠取代嗍化合物, 使用癸基三甲基溴化铵 作为表面活性剂增溶后, 可溶于磷酸缓冲液(PBS). 多 巴胺的加入可使 HMPB 的荧光显著改变, 利用其不同 波长发光强度的比值, 即可检测体系中多巴胺的浓度.

\section{2 结果与讨论}

\section{1 探针的合成}

HMPB 的合成主要分为两步(图 1): 先利用 2-溴均 三甲苯与硼酸三甲酯反应生成米基硣酸二甲酯 $(\mathrm{MOME})^{[12]}$; 再利用 1 -溴萠在乙醚溶液中与正丁基锂反 应生成有机锂化物, 加入 MOME 后即可制得 HMPB. HMPB 通过了核磁共振和质谱表征(图 S3).
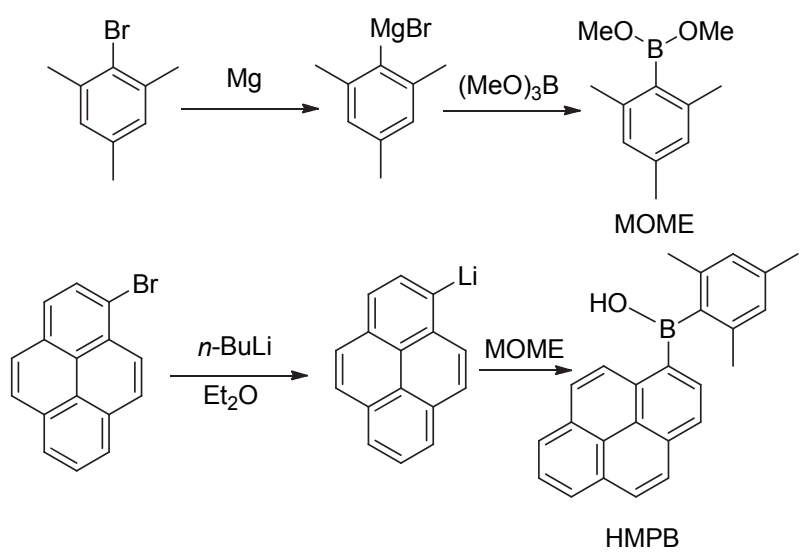

图式 1 HMPB 合成过程

Scheme 1 Synthesis of HMPB

\section{2 探针在缓冲溶液中对多巴胺的荧光响应}

由于探针 HMPB 是油溶性化合物, 在 PBS ( $\mathrm{pH} 7.4)$ 中溶解度很低, 因此, 选用癸基三甲基溴化铵作为表面 活性剂, 以增强苂光探针在 PBS 中的溶解度. 表面活性 剂的浓度会引起检测效果不同, 经过篎选得到当表面活 性剂最终的浓度为 $0.14 \mathrm{~mol} / \mathrm{L}$ 时苂光探针检测多巴胺具 有最好的效果. 未加入多巴胺时, 浓度为 $10 \mu \mathrm{mol} / \mathrm{L}$ HMPB 的 PBS 溶液在 $484 \mathrm{~nm}$ 处的苂光强度高于 $388 \mathrm{~nm}$
处; 而加入最终浓度为 $2 \mu \mathrm{mol} / \mathrm{L}$ 的多巴胺后, $388 \mathrm{~nm}$ 处 苂光强度逐渐增强, $484 \mathrm{~nm}$ 处苂光强度逐渐减弱, 10 $\min$ 内, 光谱变化基本达到饱和, $388 \mathrm{~nm}$ 处苂光强度不 再增加(图 S1), 和 $484 \mathrm{~nm}$ 处苂光强度的比值增加了 7 倍左右(图 1). 因此, 可以通过反应前后荧光的变化来检 测多巴胺. 上述过程中 HNPB 的吸收光谱基本没有变化 (图 S2).

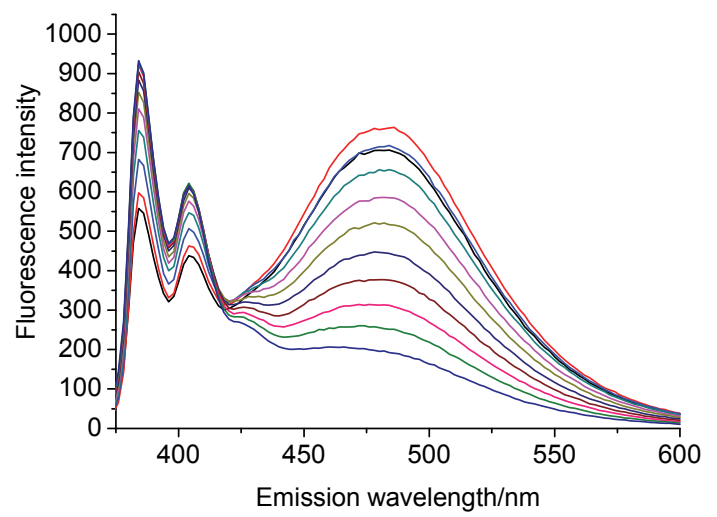

图 1 加入 $\mathrm{DA}(2 \mu \mathrm{mol} / \mathrm{L})$ 后 $0 \sim 10 \mathrm{~min}$ 时间内 $\mathrm{HMPB}(10 \mu \mathrm{mol} / \mathrm{L})$ 的发

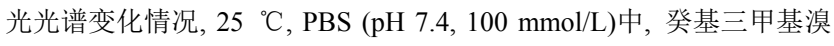
化铵 $(0.14 \mathrm{~mol} / \mathrm{L})$ 为表面活性剂, $\lambda_{\mathrm{ex}}=365 \mathrm{~nm}$

Figure 1 Time-dependent fluorescent spectral changes of $10 \mu \mathrm{mol} / \mathrm{L}$ HMPB after the addition of $2 \mu \mathrm{mol} / \mathrm{L}$ DA in $0 \sim 10 \mathrm{~min}$. Data were acquired at $25{ }^{\circ} \mathrm{C}$ in $100 \mathrm{mmol} / \mathrm{L}$ PBS (pH 7.4) with decyltrimethylammonium bromide $(0.14 \mathrm{~mol} / \mathrm{L})$ as a surfactant. $\lambda_{\mathrm{ex}}=365 \mathrm{~nm}$.

\section{3 探针的可能检测机理}

萠基非常容易形成激基缔合物，在癸基三甲基溴化 铵胶束中, HMPB 的萠基有一部分以激基缔合物的形式 存在. 故癸基三甲基溴化铵增溶的 HMPB PBS 溶液的 苂光光谱呈现两组发光峰(图 2), 长波长的发光可归因 于萠的激基缔合物发光, 短波长的发光可归因于萠的单 体发光. HMPB 与多巴胺的反应如 Eq. 1 所示. HMPB 与 多巴胺的反应产物 HMPB-DA 的溶解性水溶性优于 HMPB，萠基的激基缔合物逐渐解离生成萠基单体. 因 此, 随着多巴胺的加入, HMPB 的发光光谱长波长峰逐 渐减弱, 短波长峰逐渐升高.

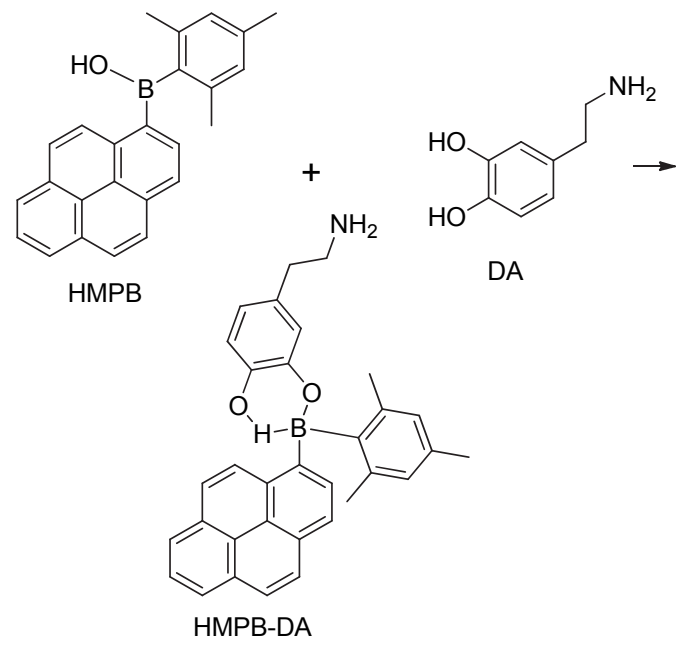




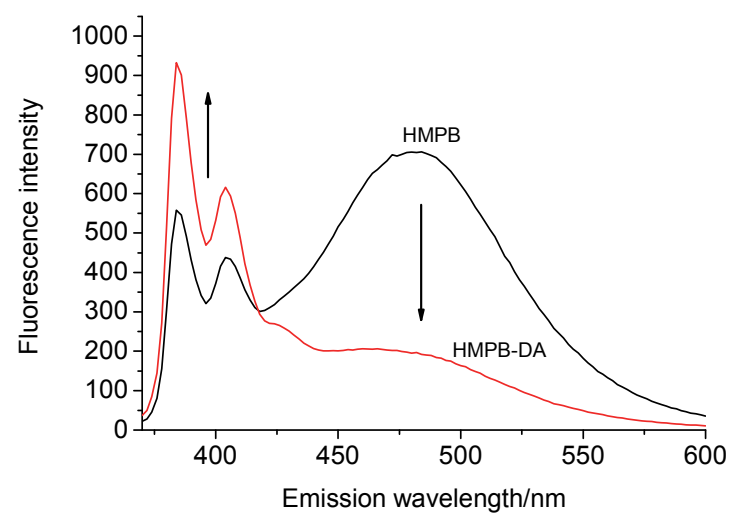

图 2 加入 $\mathrm{DA}$ 前、后 $\mathrm{HMPB}(10 \mu \mathrm{mol} / \mathrm{L})$ 的苂光光谱, $\mathrm{PBS}(\mathrm{pH}$ 7.4)中, 癸基三甲基溴化铵 $(0.14 \mathrm{~mol} / \mathrm{L})$ 为表面活性剂, $\lambda_{\mathrm{ex}}=365 \mathrm{~nm}$

Figure 2 Fluorescence spectra of HMPB $(10 \mu \mathrm{mol} / \mathrm{L})$ before and after addition of DA. Data were acquired at $25{ }^{\circ} \mathrm{C}$ in $100 \mathrm{mmol} / \mathrm{L}$ PBS $(\mathrm{pH}$ 7.4) with decyltrimethylammonium bromide $(0.14 \mathrm{~mol} / \mathrm{L})$ as a surfactant. $\lambda_{\mathrm{ex}}=365 \mathrm{~nm}$.

\section{4 探针对多巴胺的定量检测}

由图 3 可知, 在 $10 \mathrm{~min}$ 时, $10 \mu \mathrm{mol} / \mathrm{L}$ HMPB 对不同 浓度的多巴胺有不同的苂光响应. 在 $10 \sim 600 \mathrm{nmol} / \mathrm{L}$ 的 范围内, 随着多巴胺浓度增加, 388 和 $484 \mathrm{~nm}$ 处荧光强 度的比值 $\left(I_{388 \mathrm{~nm}} / I_{484 \mathrm{~nm}}\right)$ 不断增加. 同时在 $10 \sim 600$ $\mathrm{nmol} / \mathrm{L}$ 的范围内, $I_{388 \mathrm{~nm}} / I_{484 \mathrm{~nm}}$ 与多巴胺浓度间呈良好的 线性关系, 这一浓度范围与生物体内多巴胺浓度范围匹 配. 通过计算可以得到探针 HMPB 对多巴胺的检出限 为 $14.6 \mathrm{nmol} / \mathrm{L}$, 与现有报道对比, 该值为较低水平. 因 此, HMPB 的适用浓度范围和灵敏度都满足生物实验的 需求.

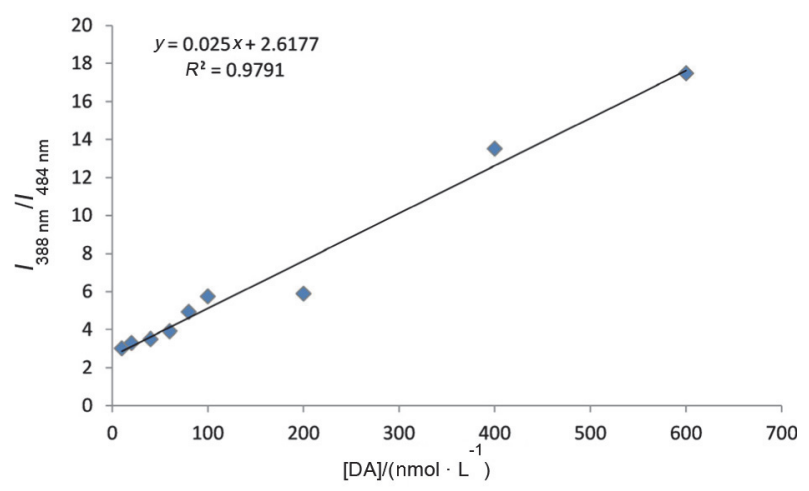

图 3 加入不同浓度 $\mathrm{DA}(10 \sim 600 \mathrm{nmol} / \mathrm{L}) 10 \mathrm{~min}$ 后 HMPB (10 $\mu \mathrm{mol} / \mathrm{L})$ 的 $I_{388 \mathrm{~nm}} / I_{484 \mathrm{~nm}}$ 与 DA 浓度的关系. $25{ }^{\circ} \mathrm{C}, \mathrm{PBS}(\mathrm{pH} 7.4,100$ $\mathrm{mmol} / \mathrm{L})$ 中, 癸基三甲基澳化铵 $(0.14 \mathrm{~mol} / \mathrm{L})$ 为表面活性剂, $\lambda_{\mathrm{ex}}=365$ $\mathrm{nm}$

Figure 3 Concentration-dependent fluorescence spectral changes of 10 $\mu \mathrm{mol} / \mathrm{L}$ HMPB after the addition of $10 \sim 600 \mathrm{nmol} / \mathrm{L}$ DA in $10 \mathrm{~min}$ : relationship between $I_{388 \mathrm{~nm}} / I_{484 \mathrm{~nm}}$ of HMPB and concentration of DA added. Data were acquired at $25{ }^{\circ} \mathrm{C}$ in $100 \mathrm{mmol} / \mathrm{L}$ PBS (pH 7.4) with decyltrimethylammonium bromide $(0.14 \mathrm{~mol} / \mathrm{L})$ as a surfactant. $\lambda_{\mathrm{ex}}=365$ $\mathrm{nm}$

\section{5 探针的选择性}

生物体内含有多种与多巴胺类似的化合物, 可能对 HMPB 的检测过程产生干扰. 因此我们选取了生物体内 的氨基酸、谷胱甘肽、葡萄糖、ATP、儿茶酚胺、阴阳 离子等, 测试 HMPB 对这些干扰物质的响应性. 当添加 的干扰物质浓度达到 $0.5 \mathrm{mmol} / \mathrm{L}$ ( $L$-DOPA、去甲肾上腺 素浓度为 $10 \mu \mathrm{mol} / \mathrm{L}$ ), HMPB 的荧光光谱对除了去甲肾 上腺素外其他干扰物未发生显著变化. 而除了特殊的研 究对象(如肾上腺), 生物体内多巴胺的浓度是去甲肾上 腺素的浓度的几十倍，甚至几百倍，在这种情况下可以 忽略去甲肾上腺素在多巴胺检测过程中的干扰. 因此, HMPB 对多巴胺表现出具有较高的选择性.

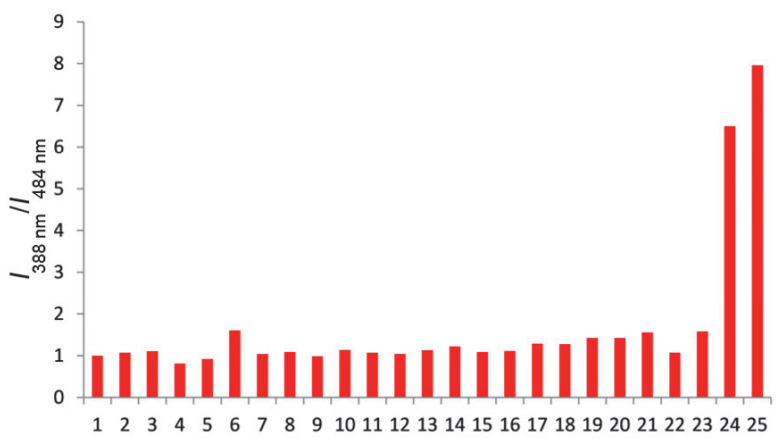

图 4 加入 $\mathrm{DA}(2 \mu \mathrm{mol} / \mathrm{L})$ 和不同干扰物质 $(0.5 \mathrm{mmol} / \mathrm{L})$ (左旋多巴和去 甲基肾上腺素浓度为 $10 \mu \mathrm{mol} / \mathrm{L}) 10 \mathrm{~min}$ 后, HMPB 的 $I_{388 \mathrm{~nm}} / I_{484 \mathrm{~mm}}$. $25{ }^{\circ} \mathrm{C}, \mathrm{PBS}(\mathrm{pH} 7.4,100 \mathrm{mmol} / \mathrm{L})$ 中, 癸基三甲基溴化铵 $(0.14 \mathrm{~mol} / \mathrm{L})$ 为 表面活性剂, $\lambda_{\mathrm{ex}}=365 \mathrm{~nm}$

Figure $4 I_{388 \mathrm{~nm}} / I_{484 \mathrm{~nm}}$ of HMPB after addition of DA $(2 \mu \mathrm{mol} / \mathrm{L})$ and interference reagents $(0.5 \mathrm{mmol} / \mathrm{L})$ in $10 \mathrm{~min}$. Data were acquired at $25{ }^{\circ} \mathrm{C}$ in $100 \mathrm{mmol} / \mathrm{L}$ PBS (pH 7.4) with decyltrimethylammonium bromide $(0.14 \mathrm{~mol} / \mathrm{L})$ as a surfactant. $\lambda_{\mathrm{ex}}=365 \mathrm{~nm}$

1. Blank control, 2: Arg. 3: His. 4: Lys. 5: Ser. 6: Gln. 7: Cys. 8: Tyr. 9: Trp. 10: Gly. 11: Hcy. 12: Glu. 13: GSH. 14: glucose. 15: KI. 16: NaCl. 17: $\mathrm{MgCl}_{2}$. 18: $\mathrm{CaCl}_{2}$. 19: BSA. 20: AA. 21: ATP. 22: ADP. 23: $L$-DOPA $(10 \mu \mathrm{mol} / \mathrm{L}) \cdot 24$ : $\mathrm{NE}(10 \mu \mathrm{mol} / \mathrm{L}) .25$ : DA.

\section{6 探针在尿液体系中对多巴胺的响应}

生命体液中的多巴胺浓度是许多疾病的诊断指标, 接下来我们研究了在生命体液中 HMPB 对多巴胺的响 应. 我们选取了含有一定量多巴胺的尿液作为检测体 系. 首先在尿液体系中加入含有表面活性剂的 HMPB 溶液, 然后再加入多巴胺水溶液, 检测 $20 \mathrm{~min}$ 内 HMPB 苂光光谱变化. 实验结果显示加入多巴胺前后, $I_{388 \mathrm{~nm}} / I_{484} \mathrm{~nm}$ 变化了 2 倍左右(图 5). 相比在缓冲溶液中, 在尿液中 HMPB 对多巴胺响应比较缓慢, 其中可能的 原因是尿液中含有的化学物质，也可以起到表面活性剂 的作用, 造成 HMPB 与多巴胺的反应比较慢. 尽管如 此, 实验结果说明 HMPB 可以在生命体液中检测外源 多巴胺.

\section{3 结论}

本文设计合成了用于检测多巴胺的比例苂光探针 


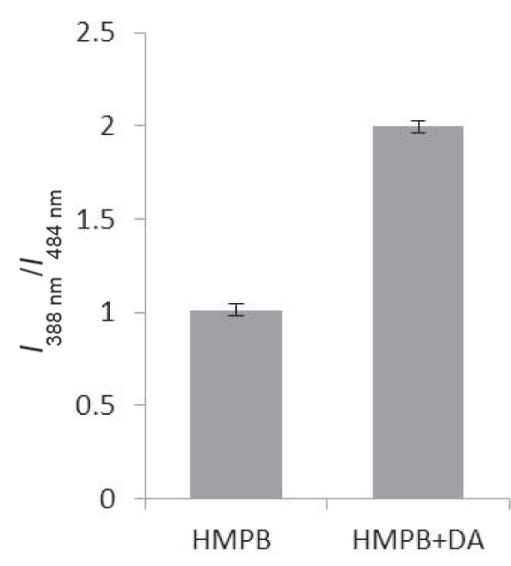

图 5 加入 DA $(10 \mu \mathrm{mol} / \mathrm{L})$ 后 $20 \mathrm{~min}$ 时间内 $\mathrm{HMPB}(10 \mu \mathrm{mol} / \mathrm{L})$ 的 $I_{388 \mathrm{~nm}} / I_{484 \mathrm{~nm}}$ 变化情况, 尿液中, 癸基三甲基溴化铵 $(0.14 \mathrm{~mol} / \mathrm{L})$ 为表面 活性剂, $\lambda_{\mathrm{ex}}=365 \mathrm{~nm}$

Figure $5 I_{388 \mathrm{~nm}} / I_{484 \mathrm{~nm}}$ changes of $10 \mu \mathrm{mol} / \mathrm{L}$ HMPB after the addition of $10 \mu \mathrm{mol} / \mathrm{L} \mathrm{DA}$ in $20 \mathrm{~min}$ and in urine with decyltrimethylammonium bromide $(0.14 \mathrm{~mol} / \mathrm{L})$ as a surfactant, $\lambda_{\mathrm{ex}}=365 \mathrm{~nm}$

HMPB，并利用表面活性剂使其应用于 PBS 缓冲溶液 中. HMPB 可与多巴胺反应生成水溶性更好的产物 HMPB-DA，此过程中 HMPB 的萠基激基缔合物逐渐解 离, 故 HMPB 对多巴胺呈现显著的比例荧光响应, 可在 10 min 内完成检测，且呈现明显的浓度效应，可在 10 $600 \mathrm{nmol} / \mathrm{L}$ 范围内检测多巴胺的浓度, 检出限低达 14.6 $\mathrm{nmol} / \mathrm{L}$. HMPB 对生物体内多种潜在干扰物都没有荧光 响应，呈现出对多巴胺的较强的选择性. 同时可以在生 命体液尿液中检测多巴胺，因此，HMPB 是一种高效、 灵敏、准确的多巴胺比例苂光探针, 为在生物体内检测 多巴胺提供了一种潜在的、有效的方法.

\section{4 实验部分}

MOME 的合成见参考文献[1]. HMPB 的合成: 氩气

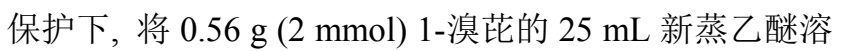
液冷却至 $-78{ }^{\circ} \mathrm{C}$, 然后缓慢滴入正丁基锂溶液(1.6 $\mathrm{mol} / \mathrm{L}, 2.5 \mathrm{~mL}, 4 \mathrm{mmol})$, 滴加完毕后, 低温反应 $1 \mathrm{~h}$, 升 至室温, 继续摚拌 $2 \mathrm{~h}$. 然后再次降温到 $-78{ }^{\circ} \mathrm{C}$, 滴入 MOME 乙醚溶液 $0.28 \mathrm{~g}(1 \mathrm{mmol})$, 滴加完毕后反应 30 $\mathrm{min}$, 升至室温, 搅拌过夜. 然后加入 $20 \mathrm{~mL}$ 稀盐酸, 继 续搅拌 $0.5 \mathrm{~h}$. 用二氯甲烷萃取 3 次, 收集有机相, 抽干 溶剂后用石油醚重结晶, 得到黄色晶体 $0.67 \mathrm{~g}, 1.9$ $\mathrm{mmol}$, 产率为 $96 \% .{ }^{1} \mathrm{H} \mathrm{NMR}\left(400 \mathrm{MHz}, \mathrm{CDCl}_{3}\right) \delta: 9.214$ (d, $J=12 \mathrm{~Hz}, 1 \mathrm{H}), 8.302(\mathrm{~d}, J=10 \mathrm{~Hz}, 1 \mathrm{H}), 8.268$ (t, $J=8$ $\mathrm{Hz}, 2 \mathrm{H}), 8.197$ (t, $J=8 \mathrm{~Hz}, 2 \mathrm{H}), 8.140(\mathrm{~d}, J=8.0 \mathrm{~Hz}, 1 \mathrm{H})$, 8.09 (d, $J=8 \mathrm{~Hz}, 1 \mathrm{H}), 8.062$ (t, $J=12 \mathrm{~Hz}, 1 \mathrm{H}), 6.975$ (s, 2H), 6.346 (s, 1H), 2.417 (s, 3H), 2.332 (s, 6H); ${ }^{13} \mathrm{C}$ NMR $\left(100 \mathrm{MHz}, \mathrm{CDCl}_{3}\right) \delta: 138.27,136.19,135.72,133.80$, $131.13,130.64,128.87,127.56,127.42,125.73,125.44$, $125.40,124.73,124.63,124.27,22.20,21.25$. GC-MS $m / z$ calcd: 348.17 , found: 348 .

\section{References}

[1] (a) Robinson, D. L.; Hermans, A.; Seipel, A. T.; Wightman, R. M. Chem. Rev. 2008, 108, 2554; (b) Hyman, S. E.; Malenka, R. C. Nat. Rev. Neurosci. 2001, 2, 695.

[2] Li, B.-R.; Hsieh, Y.-J.; Chen, Y.-X.; Chung, Y.-T.; Pan, C.-Y.; Chen, Y.-T. J. Am. Chem. Soc. 2013, 135, 16034

[3] Li, L.; Liu, H.; Shen, Y.; Zhang, J.; Zhu, J. J. Anal. Chem. 2011, 83, 661.

[4] (a) Tyagi, P.; Postetter, D.; Saragnese, D.; Randall, C.; Mirski, M.; Gracias, D. Anal. Chem. 2009, 81, 9979; (b) Koehne, J. E.; Marsh, M.; Boakye, A.; Douglas, B.; Kim, I. Y.; Chang, S.-Y.; Jang, D.-P.; Bennet, K. E.; Kimble, C.; Andrews, R. Analyst 2011, 136, 1802; (c) Zhou, J.; Wang, W.; Yu, P.; Xiong, E.; Zhang, X.; Chen, J. RSC. Adv 2014, 4, 52250; (d) Liu, Q.; Zhu, X.; Huo, Z.; He, X.; Liang, Y.; Xu, M. Talanta 2012, 97, 557; (e) Wei, X.; Chang, C.; Li, J. Acta. Chim. Sinica 2013, 71, 951 (in Chinese). (魏小平, 常川, 李建平, 化学学 报, 2013, 71,951.)

[5] Zhao, Y.; Zhao, S.; Huang, J.; Ye, F. Talanta 2011, 85, 2650.

[6] Liu, L.; Li, S.; Liu, L.; Deng, D.; Xia, N. Analyst 2012, 137, 3794.

[7] (a) Lee, H.-C.; Chen, T.-H.; Tseng, W.-L.; Lin, C.-H. Analyst 2012 137, 5352; (b) Su, H.; Sun, B.; Chen, L.; Xu, Z.; Ai, S. Anal. Methods 2012, 4, 3981.

[8] Nikolajsen, R. P.; Hansen, Å. M. Anal. Chim. Acta 2001, 449, 1.

[9] Zhang, L.; Teshima, N.; Hasebe, T.; Kurihara, M.; Kawashima, T. Talanta 1999, 50, 677.

[10] (a) Ma, Y.; Yang, C.; Li, N.; Yang, X. Talanta 2005, 67, 979; (b) Mu, Q.; Xu, H.; Li, Y.; Ma, S.; Zhong, X. Analyst 2014, 139, 93; (c) Zhao, D.; Song, H.; Hao, L.; Liu, X.; Zhang, L.; Lv, Y. Talanta 2013 107, 133; (d) Yu, C.; Luo, M.; Zeng, F.; Zheng, F.; Wu, S. Chem. Commun. 2011, 47, 9086.

[11] (a) Jackowska, K.; Krysinski, P. Anal. Bioanal. Chem. 2013, 405 , 3753; (b) She, G.; Huang, X.; Jin, L.; Qi, X.; Mu, L.; Shi, W. Small 2014, 10, 4685 .

[12] Feng, J.; Tian, K.; Hu, D.; Wang, S.; Li, S.; Zeng, Y.; Li, Y.; Yang, G. Angew. Chem., Int. Ed. 2011, 50, 8072.

(Qin, X.) 